Check for updates

Cite this: RSC Adv., 2017, 7, 18775

Received 20th February 2017

Accepted 17th March 2017

DOI: $10.1039 / c 7 r a 02086 d$

rsc.li/rsc-advances

\section{Electrospun poly- $\varepsilon$-caprolactone scaffold modified with catalytic nitric oxide generation and heparin for small-diameter vascular graft}

\author{
Jingchen Gao, Yaping Wang, Siyuan Chen, Di Tang, Li Jiang, Deling Kong (D) \\ and Shufang Wang (D) *
}

Vascular grafts are significantly needed in peripheral vascular surgery; however, small diameter grafts are not always available, and synthetic grafts perform poorly because of acute thrombosis and neointimal proliferation after implantation. This study used electrospun poly- $\varepsilon$-caprolactone (PCL) as the matrix material to build a small-diameter vascular graft. Organoselenium modified polyethyleneimine (SePEI), which can catalyze the production of nitric oxide from S-nitrosothiols, and heparin (Hep) were introduce through layer-by-layer (LbL) assembly in order to prepare a new vascular graft with improved histocompatibility and biological function. Static water contact angle measurement showed that SePEI and Hep had improved the hydrophilicity of the material, which is desirable for an active vascular graft. The results showed that the mechanical property and histocompatibility of the SePEI/Hep loaded material could meet the demands of vascular grafts. The results of cellular experiments showed that the $\mathrm{SePEI} / \mathrm{Hep}$ loaded material could promote the proliferation and adhesion of endothelial cells, which is beneficial to the rapid endothelialization of vascular grafts. And this material could inhibit the adhesion and spreading of smooth muscle cells, which can prevent the post-implantation restenosis. The SePEI/ Hep loaded material could inhibit the activation of macrophages, which is very important in reducing inflammation following graft implantation.

\section{Introduction}

Cardiovascular disease has become a threat to human health, and vascular transplantation is the most effective means of treatment for cardiovascular disease. ${ }^{\mathbf{1 - 4}}$ As a result, there is a significant need for vascular grafts in peripheral vascular surgery; however, small diameter grafts are not always available, and synthetic grafts perform poorly because of acute thrombosis and neointimal proliferation after implantation..$^{5-7}$

Macrophages are major regulators of vascularization, through the activity of different types of macrophages display different cell populations with distinct functions. ${ }^{8-10}$ Macrophages generally fall into two phenotypes: the pro-inflammatory M1 and the anti-inflammatory M2 state. The M1 (classically activated) macrophage phenotype, promotes pathogen killing and is associated with classic signs of active inflammation, particularly with chronic inflammation. The M2 (alternatively activated) macrophage phenotype, promotes immunoregulation tissue repair and constructive tissue remodeling. ${ }^{9-11}$

State Key Laboratory of Medicinal Chemical Biology, Key Laboratory of Bioactive Materials for Ministry of Education, College of Life Sciences, Institute of Molecular Biology, Nankai University, Tianjin 300071, China. E-mail: wangshufang@nankai. edu.cn
NO is an endothelium derived relaxing factor, which can inhibit the adhesion and activation of platelets, reduce inflammation and intimal hyperplasia, and promote endothelial cell (EC) growth. ${ }^{2,12-14}$ There have been plenty of researches aimed at developing vascular grafts with NO release or generation and better anti-thrombotic property and biological function. NO releasing material with blended or bounded NO donors (mainly diazeniumdiolate and S-nitrosothiols) exhibits shortages such as burst release, limited storage and toxicity caused by leaching of donors. ${ }^{15}$ More and more people turn their eyes to NO generating materials. Jun Yang et al. assembled a NO generating surface via LbL deposition of sodium alginate (Alg) and organoselenium modified polyethyleneimine (SePEI) on quartz and polymeric substrates. The immobilized SePEI species were capable of catalytically decomposing S-nitrosothiol species (RSNO) to NO in the presence of thiol reducing agents. ${ }^{16}$ Bong Kyun Oh et al. examined an approach potentially useful for the development of more thromboresistant polymeric materials based on the catalytic generation of $\mathrm{NO}$ via $\mathrm{Cu}(\mathrm{I})$ mediated reduction of nitrite ions. ${ }^{17}$ Nan Huang et al. immobilized selenocystamine on $\mathrm{TiO}_{2}$ film deposited on silicon wafer and 316 stainless steel stents for catalytic generation of NO. ${ }^{18}$

Heparin (Hep) has been widely used as an anticoagulant coating of vascular grafts. ${ }^{\mathbf{1 9 - 2 2}}$ Numerous studies have also shown that Hep can inhibit migration and proliferation of 
smooth muscle cells (SMCs) ${ }^{23,24}$ Besides, an appropriate dosage of Hep can selectively enhance EC but inhibit SMC proliferation. ${ }^{25,26}$ Additionally, it has been reported that Hep can be used as an anti-inflammatory drug in various models of inflammatory diseases. ${ }^{25,27}$ This means that a heparinized surface with adequate drug density and release kinetics will inhibit thrombosis and restenosis but will not harm the endothelium. ${ }^{22}$

Polycaprolactone (PCL) is a commonly used candidate material for small diameter artificial blood vessels. ${ }^{28-33}$ PCL is used as the main component because of its low cost and stability in processing and storage..$^{31-33}$

In order to develop an ideal small-diameter vascular graft, many research groups have employed electrospinning to fabricate fibrous scaffolds with mechanically compliant for vascular regeneration. ${ }^{34-37}$ The graft obtained through electrospinning can be tailored to closely resemble the structure and function of the native extracellular matrix (ECM) in order to facilitate cellmaterial interactions. ${ }^{37}$

In this contribution, we used PCL as the matrix material, then SePEI and Hep were introduced through LbL assembly in order to build a novel vascular graft with in situ NO generation. The ability of this material to generate NO was evaluated. The adhesion of EC, SMC and macrophage, spreading of SMC and activation of macrophage were observed. The vascular grafts made from this material were implanted in rats to assess the host tissue response. We hypothesized that this graft has the ability to catalyze NO generation, enhance EC but inhibit SMC proliferation and regulate macrophage activation.

\section{Materials and methods}

\subsection{Materials}

Poly- $\varepsilon$-caprolactone (PCL, $M_{\mathrm{w}} 80000$ ) was obtained from Solvay Interox Ltd, UK. Poly diallyldimethylammonium chloride (PDDA, $M_{\mathrm{w}} 100000-200000,20 \mathrm{wt} \%$ in water) and polyethylenimine (PEI, $M_{\mathrm{w}} 25000$ ) were purchased from SigmaAldrich. Griess Assay Kit was obtained from Beyotime Institute of Biotechnology.

\subsection{Scaffold preparation}

PCL fibrous scaffolds were prepared by electrospinning. Briefly, $12 \%(\mathrm{w} / \mathrm{v})$ PCL solution was prepared by dissolving PCL in the mixture of methanol and chloroform $(1: 5 \mathrm{v} / \mathrm{v})$. The prepared solution was fed through a syringe pump at a flow rate of $2 \mathrm{~mL}$ $\mathrm{h}^{-1}$. A high voltage of $18 \mathrm{kV}$ was supplied by a high voltage generator (DWP503-1AC, Dong-Wen High Voltage Power Supply Factory, Tianjin, China) and the distance between the needle tip (21-G) and the collector was $20 \mathrm{~cm}$. The collectors for collecting films (a steel drum, diameter $=4.7 \mathrm{~cm}$ ) and tubular grafts (a steel rod, diameter $=2 \mathrm{~mm}$ ) were self-designed and made. Syringe pump (74 900-05) from Cole Parmer was used in electrospinning, and the collectors for electrospun films were selfdesigned and made. In addition, the temperature was around $25{ }^{\circ} \mathrm{C}$ and relative humidity was around $50 \%$. The film was dried in vacuum to evaporate the residual solvent at room temperature for $48 \mathrm{~h}$.

\subsection{Surface modification by LbL assembly}

SePEI were synthesized according to reported method..$^{38,39}$ The surface modification of PCL electrospun film with SePEI and Hep was performed by LbL assembly. Briefly, PDDA, SePEI, PEI and Hep were dissolved in distilled water to make $1 \mathrm{mg} \mathrm{mL}$ solutions. A PDDA/Hep bilayer was loaded as a precursor layer, and then SePEI and Hep were deposited until a certain number of bilayers ((SePEI/Hep $\left.)_{n}\right)$ were reached. For each layer, the film was dipped into the solution for $10 \mathrm{~min}$ and then rinsed with water and blown dry by air. PEI was used as control group instead of SePEI.

\subsection{SEM observation}

The micromorphology of the materials before and after modification was observed by scanning electron microscope (SEM; QUANTA 200; FEI, USA) with an acceleration voltage of $15 \mathrm{kV}$. Samples were gold-coated before SEM.

\subsection{In vitro catalytic NO generation experiment}

S-Nitrosoglutathione (GSNO) was synthesized as described in literature and slightly modified: $:^{38,40} 2 \mathrm{~mL}$ of $1 \mathrm{M} \mathrm{HCl}$ was added to $2 \mathrm{~mL}$ of $100 \mathrm{mM}$ reduced glutathione (GSH) on ice, in dark and while stirring, followed by $2 \mathrm{~mL}$ of $100 \mathrm{mM} \mathrm{NaNO}_{2}$, and the solution was kept in dark for $40 \mathrm{~min}$; after the reaction finished, $\mathrm{pH}$ was adjusted to 7.0 using $1 \mathrm{M}$ HCL and $1 \mathrm{M} \mathrm{NaOH}$, and the volume was made up to $10 \mathrm{~mL}$ by pure water; then the resulted solution was diluted and read on a spectrophotometer at $335 \mathrm{~nm}$, and the concentration of final GSNO was calibrated via a calibration curve according to the reported equation $A=$ $0.92 c$. $^{40}$

The PCL-(SePEI/Hep $)_{n}$ film was cut into round pieces and put into 48 wells plates, using PCL film and PCL-(PEI/Hep) $)_{n}$ as control. $300 \mu \mathrm{L}$ of working solution $(200 \mu \mathrm{M}$ GSNO, $200 \mu \mathrm{M} \mathrm{GSH}$ and 2 mM EDTA in Phosphate Buffer solution) was added into each well, and the plates were kept in dark for certain times. After that, the catalytic NO generation was measured using a Griess Assay Kit.

\subsection{Static water contact angle measurement}

Electrospun film was put on a glass slide and the water contact angle was measured. Images were shot using SPCA (HARKE, Beijing, China) and determined using DrawTool. The average was made out of ten parallel measurements $(n=10)$.

\subsection{Mechanical strength test}

For mechanical testing, specimens (30 $\mathrm{mm} \times 10 \mathrm{~mm}, n=6)$ were prepared. Mechanical properties were determined by a tensile-testing machine (2519-104, Instron, Norwood, MA, USA) at an elongation speed of $10 \mathrm{~mm} \mathrm{~min}^{-1}$.

\subsection{The adhesion of EC}

For cellular experiments (section 2.8-2.14), sample films were cut into circular pieces, put into 48 -well tissue culture polystyrene (TCPS) plates and sterilized under ultraviolet radiation 
overnight. EC was colored by 1,1'-dioctadecyl-3,3,3', $3^{\prime}$-tetramethylindocarbocyanine perchlorate (DiI, Beyotime, China). Each well was added with $1 \times 10^{4}$ of cells. $200 \mu \mathrm{M}$ GSNO and $200 \mu \mathrm{M}$ GSH were added to the well and incubated for $4 \mathrm{~h}$ at $37{ }^{\circ} \mathrm{C}, 5 \%$ $\mathrm{CO}_{2}$. The adhesion of EC on the membranes was examined by fluorescence microscopy. Cell counting was performed with a hemocytometer.

\subsection{The proliferation of EC}

The proliferation of EC was assessed using MTT test. Each well was added with $1 \times 10^{4}$ of cells. $200 \mu \mathrm{M}$ GSNO and $200 \mu \mathrm{M} \mathrm{GSH}$ were added to the well and incubated at $37{ }^{\circ} \mathrm{C}, 5 \% \mathrm{CO}_{2}$. The medium was changed every $24 \mathrm{~h} .1$ and 3 days after seeding, 50 $\mu \mathrm{L}$ of $5 \mathrm{mg} \mathrm{mL}{ }^{-1}$ MTT solution was added to the wells and incubated for $4 \mathrm{~h}$ at $37^{\circ} \mathrm{C}$. DMSO was used to replace the solution to dissolve the formazan salts, and the final absorbance of each sample was measured using a Labsystems Multiskan RC 96-well microplate reader at $490 \mathrm{~nm}$ after incubation for $30 \mathrm{~min}$ at $37^{\circ} \mathrm{C}$.

\subsection{Platelet adhesion test}

Each well was added with $1 \times 10^{8}$ of platelets. $200 \mu \mathrm{M}$ GSNO and $200 \mu \mathrm{M}$ GSH were added to the well and incubated for $1 \mathrm{~h}$ at $37{ }^{\circ} \mathrm{C}, 5 \% \mathrm{CO}_{2}$.

According to the instructions of LDH (leakage of cytosolic lactate dehydrogenase) Kit, the absorbance read at $490 \mathrm{~nm}$ to determine LDH activity. In addition, the adhesion of platelets on the membranes was examined by SEM.

\subsection{The adhesion of SMC}

SMC was colored by DiI. Each well was added with $1 \times 10^{4}$ of cells. $200 \mu \mathrm{M}$ GSNO and $200 \mu \mathrm{M}$ GSH were added to the well and incubated for $4 \mathrm{~h}$ at $37^{\circ} \mathrm{C}, 5 \% \mathrm{CO}_{2}$. The adhesion of SMC on the membranes was examined by fluorescence microscopy. Cell counting was performed with a hemocytometer.

\subsection{The spreading of SMC}

Each well was added with $1 \times 10^{4}$ of cells. $200 \mu \mathrm{M}$ GSNO and $200 \mu \mathrm{M}$ GSH were added to the well and incubated for $4 \mathrm{~h}$ at $37^{\circ} \mathrm{C}, 5 \% \mathrm{CO}_{2}$. The spreading of SMC on the membranes was examined by SEM.

\subsection{The adhesion of macrophage}

Macrophage (RAW264.7) was colored by DiI. Each well was added with $1 \times 10^{4}$ of cells. $200 \mu \mathrm{M}$ GSNO and $200 \mu \mathrm{M}$ GSH were added to the well and incubated for $6 \mathrm{~h}$ at $37^{\circ} \mathrm{C}, 5 \% \mathrm{CO}_{2}$. The adhesion of RAW264.7 on the membranes was examined by fluorescence microscopy. Cell counting was performed with a hemocytometer.

\subsection{The spread of macrophage}

Each well was added with $1 \times 10^{4}$ of RAW264.7. $200 \mu \mathrm{M}$ GSNO and $200 \mu \mathrm{M}$ GSH were added to the well and incubated for $6 \mathrm{~h}$ at $37{ }^{\circ} \mathrm{C}, 5 \% \mathrm{CO}_{2}$. The shape of RAW264.7 was examined by SEM.

\subsection{The histocompatibility of the material}

The use of experimental animals was approved by the Animal Experiments Ethical Committee of Nankai University and carried out in conformity with the Guide for Care and Use of Laboratory Animals. Animals were anesthetized upon intraperitoneal injection of chloral hydrate $\left(300 \mathrm{mg} \mathrm{kg}^{-1}\right)$, and the hair on the back was removed. Four subcutaneous pockets on the back were made by incision. After implanting fibrous samples subcutaneously (PCL films were placed subcutaneously in the left side, PCL-(SePEI/Hep) ${ }_{10}$ films were placed subcutaneously in the right side, $n=4$ for each sample), the incision was sutured. After 1 and 2 weeks, implanted scaffolds were retrieved with surrounding tissues. All the fresh tissues attached to the matrices were removed without damage to the underlying fibrous matrices. The explants were rinsed with normal saline, and then stained by H\&E method for observation.

\subsection{Statistical analysis}

All quantitative results are reported as mean \pm standard deviation. Student's $t$-tests analysis of variance was used to compare the groups. High significance was set at $p<0.01$.

\section{Results and discussions}

\subsection{Morphology of nanofibrous mats}

SEM images of PCL electrospun nanofibrous mats are shown in Fig. 1. The fibrous morphology of the scaffolds exhibited continuous, smooth sub-micron fibers without beads. The electrospun nanofibrous mats were little changed after modification, and the outlines of polymer nanofibers were well preserved after the treatments of self-assembly. In many studies, self-assembly wouldn't seriously affect the structural features of nanofibers. ${ }^{41-43}$

\subsection{Assay of in vitro catalytic NO generation}

The result of in vitro catalytic NO generation (Fig. 2) shows that the NO generation of the experimental group (PCL-(SePEI/ Hep) $)_{n}$ ) was significantly higher than that of the control groups (TCPS, PCL and PCL-(PEI/Hep $\left.)_{n}\right)$. In addition, the NO generation increased with the increase of the layer number of assembly and the time. This catalyzing approach has a great potential in adaptability where the catalyzing ability could be adjusted through the control of quantities of assembled layers and loaded catalyst. Another advantage of this new material is that when it contacts peripheral blood that provides a constant source of NO donors there will avoid the burst release and limited storage in NO generation profile, which is a critical and inevitable crux for most NO releasing materials. In the following experiments, we used PCL-(SePEI/Hep) ${ }_{10}$ materials which had both an adequate NO generation and a fine micromorphology.

\subsection{Characterization}

As Fig. 3A shows, the contact angle of PCL was around $120^{\circ}$, while after modification, the contact angle was 

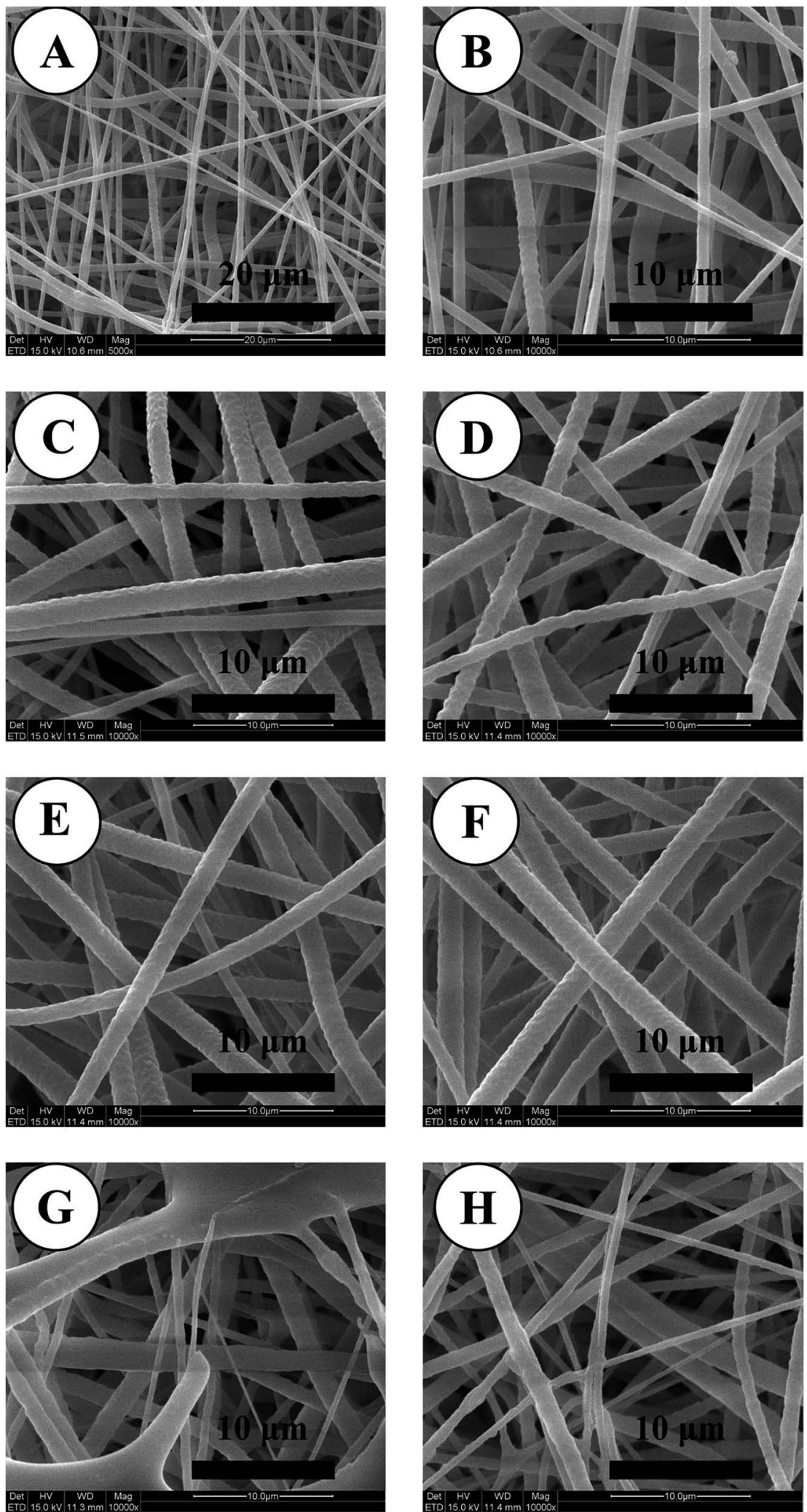

Fig. 1 Micromorphology of electrospun nanofibrous mats before and after the deposition of the polyelectrolytes (SEM images). (A and B) PCL;

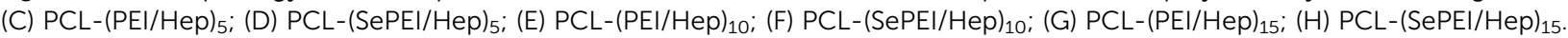

decreased to around $90^{\circ}$, showing that the assembly can enhance the hydrophilicity of the materials. PCL is hydrophobic and does not have any physiological active sites, which makes it unfavorable for cell growth when contacting with the living body, ${ }^{44}$ while the assembly can improve its hydrophilicity. 

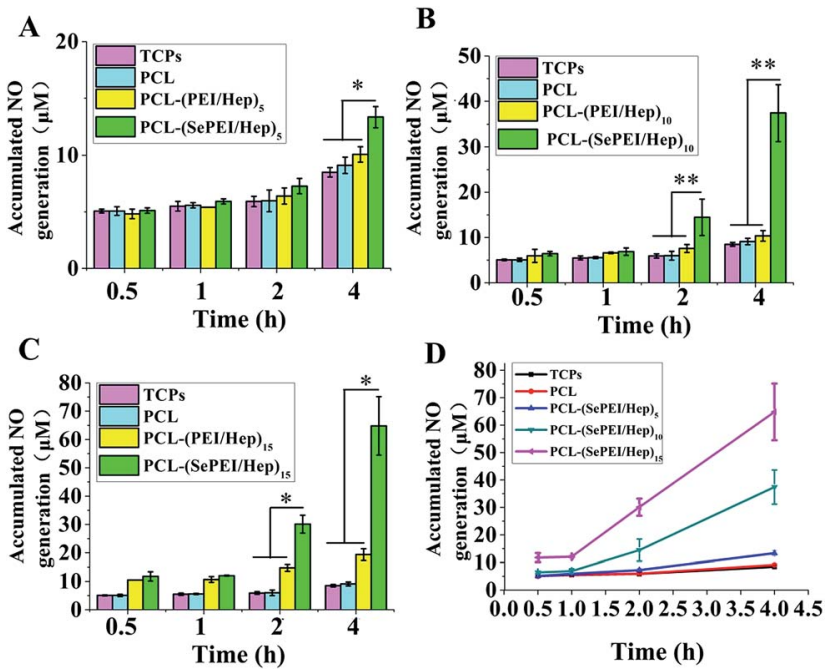

Fig. 2 Catalytic NO generation from GSNO on different nanofibrous mats $(* p<0.05 ; * * p<0.01 ; n=6)$. (A) PCL- $(\mathrm{SePEI} / \mathrm{Hep})_{5}, \mathrm{PCL}-(\mathrm{PEI} /$ Hep) 5 ; (B) PCL-(SePEl/Hep) ${ }_{10}$, PCL-(PEl/Hep) ${ }_{10}$; (C) PCL-(SePEl/Hep) $)_{15}$, PCL-(PEI/Hep $)_{15} ;$ (D) PCL-(SePEI/Hep $)_{n}$.

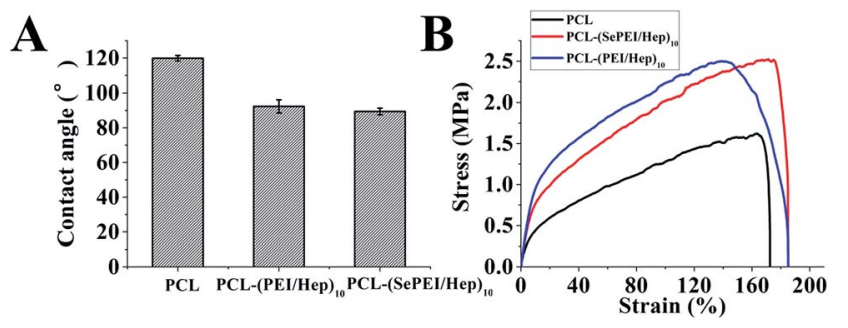

Fig. 3 Characterization of electrospun nanofibrous mats before and after the deposition of the polyelectrolytes. (A) Contact angles $(n=10)$. (B) Typical stress-strain curves $(n=6)$.

Some studies found that the optimum hydrophilic/ hydrophobic surfaces for the growth of SMC and EC were not identical; ${ }^{4546}$ and the optimum endothelialization can be achieved by moderately hydrophilic surface, which effectively inhibits the proliferation of SMC without doing harm to EC. ${ }^{\mathbf{4 7}}$ Moreover, albumin strongly adsorbed and resisted replacement by cell adhesive proteins on hydrophobic self-assembled monolayers, which effectively prohibited the cell adhesion. ${ }^{48}$ As a result, the assembly may be helpful for cell adhesion and tissue regeneration for the improvement of the hydrophilicity of PCL.

The tensile strengths of the electrospun mat were measured using a tensile tester. According to the stress-strain curves shown in Fig. 3B, the maximum stress of PCL was 1.5 MPa, and the maximum strain was about $150 \%$; those of PCL-(PEI/Hep) 10 were $2.5 \mathrm{MPa}$ and about $140 \%$ respectively; and those of PCL$(\mathrm{SePEI} / \mathrm{Hep})_{10}$ were $2.5 \mathrm{MPa}$ and about $180 \%$ respectively $(n=$ 6). The maximum stress of femoral artery was 1-2 MPa and the maximum strain was $63-76 \%{ }^{49}$ It suggested that the assembly didn't change the mechanical properties of PCL much, and its mechanical properties can meet the demands of vascular tissue engineering.

\subsection{In vitro cells culture}

The results of in vitro cells experiments are shown in Fig. 4.

Fig. 4A shows the cell attachment after incubating endothelial cell for $4 \mathrm{~h}$, while Fig. $4 \mathrm{~B}$ shows the cellular proliferation after incubating endothelial cell for 1 day and 3 day. In Fig. 4A, the image shows that ECs were attached on the mats after incubating the cells for $4 \mathrm{~h}$. These results demonstrated cellular attachment of ECs under static incubating on all the electrospun mats, and cell attachment on the PCL-(SePEI/Hep) $)_{10}$ sample was clearly better than the other two samples. Identically, in Fig. 4B, it had similar results. After 3 days, cell proliferation on the PCL-(SePEI/Hep) ${ }_{10}$ sample was clearly better than the other two samples, too. It indicated that the PCL-(SePEI/ Hep) $)_{10}$ sample was benefit for promoting the adhesion and propagation of ECs.

The result of platelet adhesion is shown in Fig. 4C. It can be seen from Fig. 4C that compared with PCL, there was less platelet adhesion on the samples after modification, especially PCL-(SePEI/Hep) $)_{10}$. Besides, the platelets which did adhere were highly spherical in morphology indicating a nonactivated state on the PCL-(SePEI/Hep) $)_{10}$ sample. It's supposed that on one hand, Hep is effective in combating coagulation, which can decrease platelet adhesion and activation; $^{22,50,51}$ on the other hand, PCL-(SePEI/Hep) ${ }_{10}$ sample can induce GSNO to release NO, which plays a key role in preventing platelet adhesion, aggregation and activation. ${ }^{2,18}$ So after assembly with SePEI and Hep, the material with good anticoagulant hemorrhagic can inhibit platelet adhesion. Earlier studies have demonstrated that NO releasing or generating materials could efficiently reduce the adhesion of platelets, ${ }^{2,18}$ and the graft constructed in this study showed similar inhibiting function.

Fig. 4D shows the cell attachment after seeding SMC for $4 \mathrm{~h}$, while Fig. 5 shows the cell morphology of SMC growth; in Fig. 4D, the image shows that SMCs were attached on the mats after incubating for $4 \mathrm{~h}$. These results demonstrated that cell attachment on the PCL-(SePEI/Hep) ${ }_{10}$ sample was clearly less than on the other two samples. In addition, in Fig. 5, cell proliferation on the PCL-(SePEI/Hep) ${ }_{10}$ sample was clearly more inhibited than the other two samples as evidenced by the development of lamellipodia, which is an indicator of cell spreading. It meant that NO catalytic effect played a more important role in inhibiting the spreading of SMCs. It indicated that the PCL-(SePEI/Hep) $)_{10}$ sample can inhibit the adhesion and spreading of SMCs.

Fig. $4 \mathrm{E}$ shows the attachment after incubating macrophages cell for 6 h, while Fig. 5 shows the morphology of macrophages on electrospun nanofibrous mats. In Fig. 4E, the image shows that macrophages were attached on the mats after seeding the cells for $6 \mathrm{~h}$. These results demonstrated that cell attachment on the PCL-(SePEI/Hep) ${ }_{10}$ sample was clearly less than the other two samples. In addition, in Fig. 5, cell proliferation on the PCL(SePEI/Hep) ${ }_{10}$ sample was smaller than the other two samples, which indicated that macrophages on the PCL-(SePEI/Hep) $)_{10}$ sample hadn't been activated. ${ }^{52,53}$ A common component of the foreign-body response to implanted materials is the presence of 
A

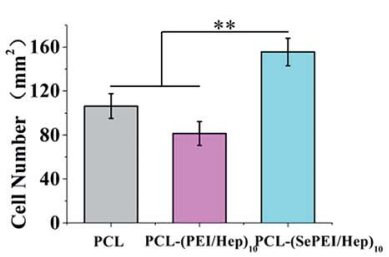

C
B

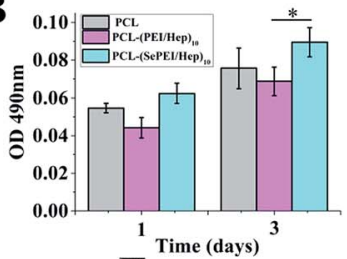

$\mathbf{E}$

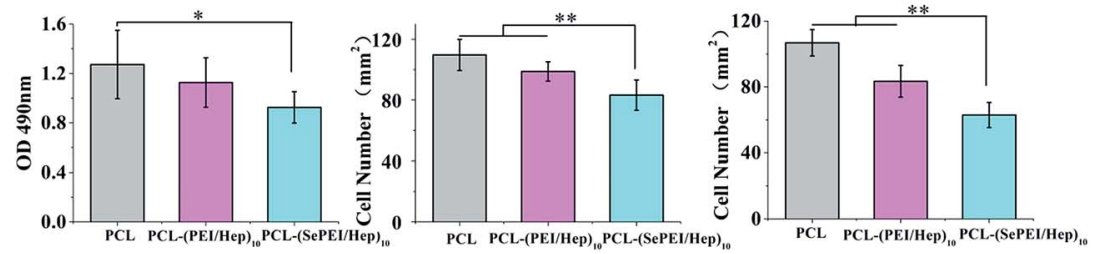

Fig. 4 Cellular adhesion and proliferation on nanofibrous mats $(* p<0.05 ; * * p<0.01)$. (A) EC adhesion counted after 4 h of incubation with GSNO and GSH $(n=6)$. (B) EC proliferation measured by MTT assay after 1 and 3 days of incubation with GSNO and GSH ( $n=6)$. (C) Platelet adhesion quantified by LDH assay after $1 \mathrm{~h}$ of incubation with GSNO and GSH $(n=6)$. (D) SMC adhesion counted after $4 \mathrm{~h}$ of incubation with GSNO and GSH $(n=6)$. (E) Macrophage adhesion counted after $6 \mathrm{~h}$ of incubation with GSNO and GSH $(n=6)$.

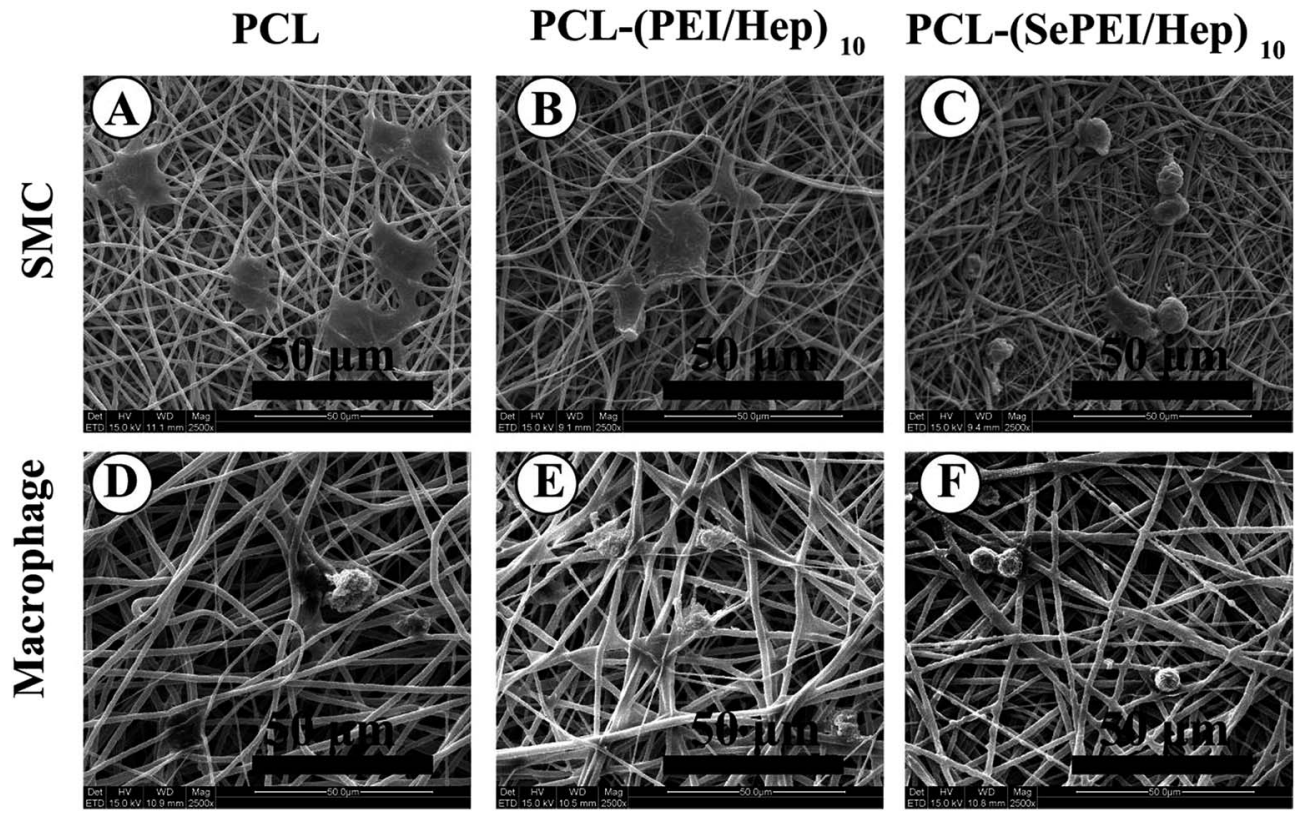

Fig. 5 Morphology of SMCs and macrophages on different samples (scale bar: $50 \mu \mathrm{m}$ ).

adherent macrophages that fuse to form foreign-body giant cells (FBGCs). It had been shown that these multinucleated cells were responsible for the damage and failure of the implant. ${ }^{54}$ The adhesion of macrophages and formation of FBGCs have been commonly observed on the surfaces of biomaterials. ${ }^{52-55}$ Results in this study meant that the PCL-(SePEI/Hep) ${ }_{10}$ sample could inhibit the adhesion and activation of macrophages, which might reduce the host foreign body response following implantation of biomaterial.

Previous studies have shown that Hep is always considered to harm ECs $;^{56}$ however, some recent studies suggested that an appropriate Hep dosage selectively enhances EC but inhibits SMC proliferation. ${ }^{22,26}$ This means that the heparinized surface with adequate drug density will inhibit thrombosis and restenosis but will not harm the endothelium. ${ }^{22}$ Moreover, NO is conducive to endothelial regeneration $;^{22}$ and it can lead to vasorelaxation, endothelial regeneration, and inhibition of leukocyte chemotaxis. ${ }^{57,58}$ These theories agreed with our experimental results. Regulated by NO and Hep, the material can promote the adhesion and propagation of EC, which is benefit for the rapid re-endothelialization of new blood vessels; the material can inhibit the spreading of SMC, which can prevent the neointimal hyperplasia. In addition, the material can affect the activation of macrophages. These results indicated that maybe the material can affect the inflammatory response to regulate the vascular remodeling. The PCL-(SePEI/Hep) $)_{10}$ sample was proved useful to the vascular remodeling in many ways. 

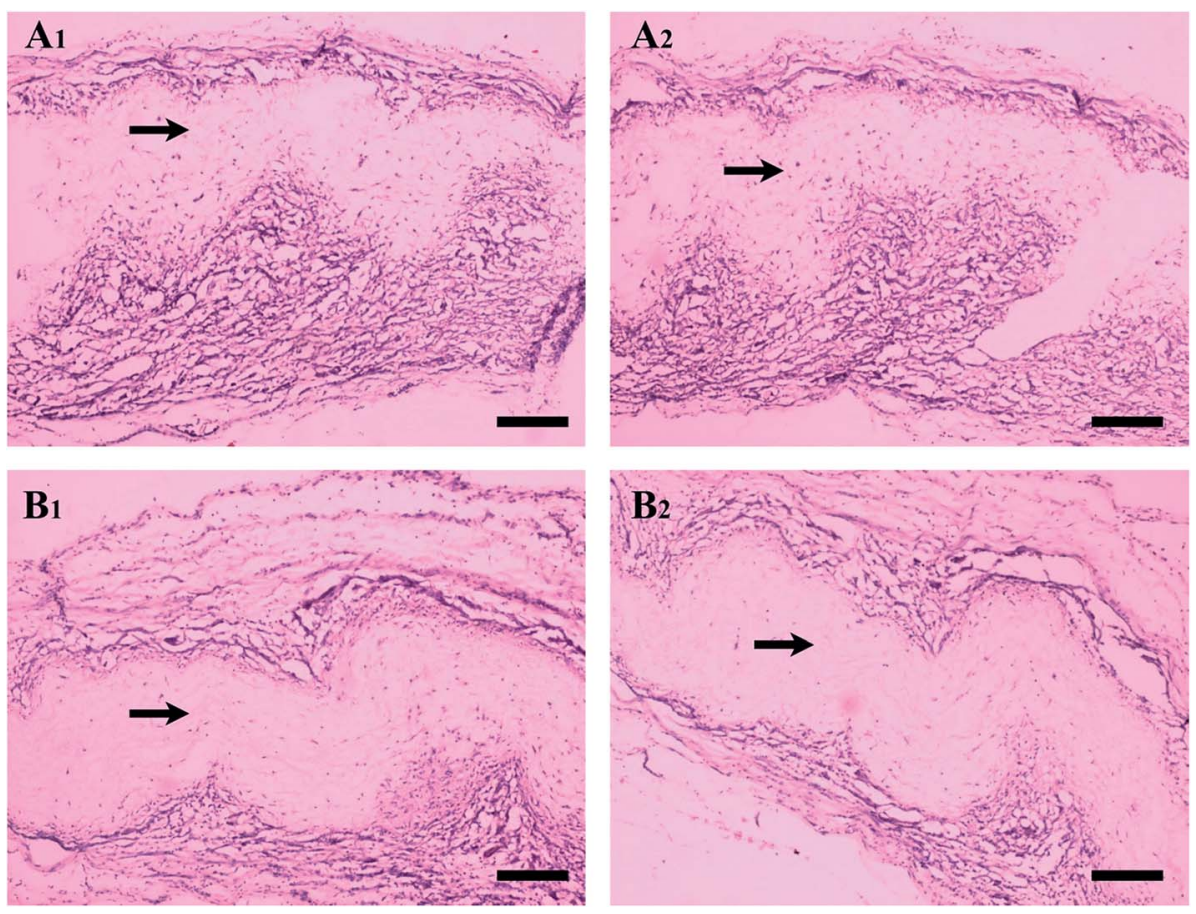

Fig. 6 In vivo biocompatibility of the nanofibrous mats (H\&E staining of samples explanted after subcutaneous implantation; scale bar: $200 \mu$ m; the black arrows indicate the mats). $\left(A_{1}\right)$ PCL, 1 week; $\left(A_{2}\right) P C L, 2$ week; $\left(B_{1}\right) P C L-(S e P E I / H e p)_{10}, 1$ week; $\left(B_{2}\right) P C L-(S e P E I / H e p)_{10}, 2$ week.

\subsection{In vivo biocompatibility of electrospun nanofibers membranes}

The 1 week post-operation is a high-incidence season of acute inflammatory response to biomaterials, which is marked by the presence of neutrophils. ${ }^{59}$ At 1 week post-operation, the acute inflammatory response to biomaterials, marked by the presence of neutrophils, usually resolves quickly. Fig. 6 shows the result of H\&E staining after subcutaneous implant test. The black arrows indicated the mats, and most of the cells stained darkly were macrophages. In Fig. 6, there was no obvious cell aggregation of neutrophils, which indicated that the materials had good biocompatibility and caused no severe acute inflammation. At 1 week after implantation, it can be seen that compared with the PCL sample; there were fewer macrophages around the PCL-(SePEI/Hep) $)_{10}$ sample. And the result of 2 week after implantation didn't change much. The result indicates that the acute inflammatory response in the PCL-(SePEI/Hep) ${ }_{10}$ sample was milder than the PCL sample. It may be due to NO can inhibit the activation of macrophages.

NO had a number of protective effects for the health of blood vessels, including inhibiting SMC proliferation and suppressing adhesion of immune cells and platelets to the endothelium..$^{58-60}$ From the results, we can see that the self-assembly materials for catalytic NO can inhibit the adhesion and activation of macrophages. Maybe NO has effect on the macrophages in other ways, such as functional polarization, a recent study has found that NO effect involves polarization of macrophages toward an antiinflammatory M2 phenotype.$^{61}$ The M2 phenotype plays a major role in tissue remodeling and suppression of inflammatory immune reactions by secreting transforming growth factor$\beta$ (TGF- $\beta$ ) and IL-10. ${ }^{10,62,63}$ The presence of such anti- inflammatory cytokines and the tissue remodeling response can aid in the vascularization of regenerative biomaterials by inhibiting fibrous tissue formation. ${ }^{10}$ This still needs further study.

\section{Conclusions}

In this study, SePEI and Hep were introduced through LbL assembly in order to build a vascular graft with in situ NO generation. The catalyzing capability was demonstrated by in vitro NO generation experiment, and the cellular compatibility was proven good in fibroblast proliferation test. In biological function evaluations, the PCL-(SePEI/Hep) $)_{10}$ sample can promote the adhesion and propagation of EC, inhibit the adhesion and spreading of SMC, inhibit the adhesion and activation of macrophages, and effected the macrophages around the materials implanted in rats. This study is novel in that we utilized the approach of catalytic NO generation to improve the blood compatibility and biological function of small-diameter vascular graft, and we made a preliminary study of the macrophages, including the adhesion and activation of macrophages, and the biocompatibility of materials implanted in rats. This would provide a promising method for bioactive materials and vascular tissue engineering research. In vivo experiments using tubular grafts built from materials described in this study are now ongoing.

\section{Acknowledgements}

This work was supported by National Natural Science Foundation of China (grant 81671842), PCSIRT (No. IRT13023), Natural 
Science Foundation of Tianjin City (grant 16JCZDJC37600) and supported by the State Key Laboratory of Medicinal Chemical Biology (201603007).

\section{References}

1 A. de Mel, G. Jell, M. M. Stevens and A. M. Seifalian, Biofunctionalization of biomaterials for accelerated in situ endothelialization: a review, Biomacromolecules, 2008, 9, 2969-2979.

2 C. M. Frendl, S. M. Tucker, N. A. Khan, M. B. Esch, S. Kanduru, T. M. Cao, et al., Endothelial retention and phenotype on carbonized cardiovascular implant surfaces, Biomaterials, 2014, 35, 7714-7723.

3 R. Y. Kannan, H. J. Salacinski, P. E. Butler, G. Hamilton and A. M. Seifalian, Current status of prosthetic bypass grafts: a review, J. Biomed. Mater. Res., Part B, 2005, 74, 570-581.

4 D. F. Williams, On the mechanisms of biocompatibility, Biomaterials, 2008, 29, 2941-2953.

5 W. Zeng, W. Yuan, L. Li, J. Mi, S. Xu, C. Wen, et al., The promotion of endothelial progenitor cells recruitment by nerve growth factors in tissue-engineered blood vessels, Biomaterials, 2010, 31, 1636-1645.

6 F. Hook, B. Kasemo, M. Grunze and S. Zauscher, Quantitative biological surface science: challenges and recent advances, ACS Nano, 2008, 2, 2428-2436.

7 T. Cyrus, S. A. Wickline and G. M. Lanza, Nanotechnology in interventional cardiology, Wiley Interdiscip. Rev.: Nanomed. Nanobiotechnol., 2012, 4, 82-95.

8 K. L. Spiller, R. R. Anfang, K. J. Spiller, J. Ng, K. R. Nakazawa, J. W. Daulton, et al., The role of macrophage phenotype in vascularization of tissue engineering scaffolds, Biomaterials, 2014, 35, 4477-4488.

9 D. P. Vasconcelos, A. C. Fonseca, M. Costa, I. F. Amaral, M. A. Barbosa, A. P. Aguas, et al., Macrophage polarization following chitosan implantation, Biomaterials, 2013, 34, 9952-9959.

10 R. Sridharan, A. R. Cameron, D. J. Kelly, C. J. Kearney and F. J. O'Brien, Biomaterial based modulation of macrophage polarization: a review and suggested design principles, Mater. Today, 2015, 18, 313-325.

11 J. S. Duffield, The inflammatory macrophage: a story of Jekyll and Hyde, Clin. Sci., 2003, 104, 27-38.

12 M. Kushwaha, J. M. Anderson, C. A. Bosworth, A. Andukuri, W. P. Minor, J. R. Lancaster Jr, et al., A nitric oxide releasing, self assembled peptide amphiphile matrix that mimics native endothelium for coating implantable cardiovascular devices, Biomaterials, 2010, 31, 1502-1508.

13 S. Hwang and M. E. Meyerhoff, Polyurethane with tethered copper(II)-cyclen complex: preparation, characterization and catalytic generation of nitric oxide from Snitrosothiols, Biomaterials, 2008, 29, 2443-2452.

14 A. de Mel, N. Naghavi, B. G. Cousins, I. Clatworthy, G. Hamilton, A. Darbyshire, et al., Nitric oxide-eluting nanocomposite for cardiovascular implants, J. Mater. Sci.: Mater. Med., 2014, 25, 917-929.
15 Y. D. Wu and M. E. Meyerhoff, Nitric oxide-releasing/ generating polymers for the development of implantable chemical sensors with enhanced biocompatibility, Talanta, 2008, 75, 642-650.

16 J. Yang, J. L. Welby and M. E. Meyerhoff, Generic nitric oxide (NO) generating surface by immobilizing organoselenium species via layer-by-layer assembly, Langmuir, 2008, 24, 10265-10272.

17 B. K. Oh and M. E. Meyerhoff, Catalytic generation of nitric oxide from nitrite at the interface of polymeric films doped with lipophilic $\mathrm{Cu}(\mathrm{II})$-complex: a potential route to the preparation of thromboresistant coatings, Biomaterials, 2004, 25, 283-293.

18 Y. Weng, Q. Song, Y. Zhou, L. Zhang, J. Wang, J. Chen, $\mathrm{N}$. Huang, et al., Immobilization of selenocystamine on $\mathrm{TiO}_{2}$ surfaces for in situ catalytic generation of nitric oxide and potential application in intravascular stents, Biomaterials, 2011, 32, 1253-1263.

19 F. Gong, X. Cheng, S. Wang, Y. Zhao, Y. Gao and H. Cai, Heparin-immobilized polymers as non-inflammatory and non-thrombogenic coating materials for arsenic trioxide eluting stents, Acta Biomater., 2010, 6, 534-546.

20 G. Li, P. Yang, W. Qin, M. F. Maitz, S. Zhou and N. Huang, The effect of coimmobilizing heparin and fibronectin on titanium on histocompatibility and endothelialization, Biomaterials, 2011, 32, 4691-4703.

21 Z. Yang, J. Wang, R. Luo, M. F. Maitz, F. Jing, H. Sun, et al., The covalent immobilization of heparin to pulsed-plasma polymeric allylamine films on 316L stainless steel and the resulting effects on histocompatibility, Biomaterials, 2010, 31, 2072-2083.

22 T. Liu, Y. Liu, Y. Chen, S. Liu, M. F. Maitz, X. Wang, et al., Immobilization of heparin/poly-(L)-lysine nanoparticles on dopamine-coated surface to create a heparin density gradient for selective direction of platelet and vascular cells behavior, Acta Biomater., 2014, 10, 1940-1954.

23 N. Cindhuchao, D. A. Quinn, H. G. Garg and C. A. Hales, Heparin inhibits SMC growth in the presence of human and fetal bovine serum, Biochem. Biophys. Res. Commun., 2003, 302, 84-88.

24 R. A. Hoshi, R. Van Lith, M. C. Jen, J. B. Allen, K. A. Lapidos and G. Ameer, The blood and vascular cell compatibility of heparin-modified ePTFE vascular grafts, Biomaterials, 2013, 34, 30-41.

25 Y. Yang, P. K. Qi, F. Wen, X. Y. Li, Q. Xia, M. F. Maitz, et al., Mussel-Inspired One-Step Adherent Coating Rich in Amine Groups for Covalent Immobilization of Heparin: Histocompatibility, Growth Behaviors of Vascular Cells, and Tissue Response, ACS Appl. Mater. Interfaces, 2014, 6, 14608-14620.

26 Z. Yang, Q. Tu, J. Wang and N. Huang, The role of heparin binding surfaces in the direction of endothelial and smooth muscle cell fate and re-endothelialization, Biomaterials, 2012, 33, 6615-6625.

27 J. P. Boyle, R. H. Smart and J. K. Shirey, Heparin In the Treatment of Chronic Obstructive Bronchopulmonary Disease, Am. J. Cardiol., 1964, 14, 25-28. 
28 S. Sarkar, G. Y. Lee, J. Y. Wong and T. A. Desai, Development and characterization of a porous micro-patterned scaffold for vascular tissue engineering applications, Biomaterials, 2006, 27, 4775-4782.

29 M. C. Serrano, R. Pagani, M. Manzano, J. V. Comas and M. T. Portoles, Mitochondrial membrane potential and reactive oxygen species content of endothelial and smooth muscle cells cultured on poly(epsilon-caprolactone) films, Biomaterials, 2006, 27, 4706-4714.

30 M. C. Serrano, M. T. Portoles, M. Vallet-Regi, I. Izquierdo, L. Galletti, J. V. Comas, et al., Vascular endothelial and smooth muscle cell culture on $\mathrm{NaOH}$-treated poly(epsiloncaprolactone) films: a preliminary study for vascular graft development, Macromol. Biosci., 2005, 5, 415-423.

31 Z. G. Tang, J. T. Callaghan and J. A. Hunt, The physical properties and response of osteoblasts to solution cast films of PLGA doped polycaprolactone, Biomaterials, 2005, 26, 6618-6624.

32 N. F. Huang, S. Patel, R. G. Thakar, J. Wu, B. S. Hsiao, B. Chu, et al., Myotube assembly on nanofibrous and micropatterned polymers, Nano Lett., 2006, 6, 537-542.

33 H. J. Wu, J. T. Fan, C. C. Chu and J. Wu, Electrospinning of small diameter 3-D nanofibrous tubular scaffolds with controllable nanofiber orientations for vascular grafts, $J$. Mater. Sci.: Mater. Med., 2010, 21, 3207-3215.

$34 \mathrm{~J}$. Wu and C. C. Chu, Block copolymer of poly(ester amide) and polyesters: synthesis, characterization, and in vitro cellular response, Acta Biomater., 2012, 8, 4314-4323.

35 C. K. Hashi, Y. Q. Zhu, G. Y. Yang, W. L. Young, B. S. Hsiao, $\mathrm{K}$. Wang, et al., Antithrombogenic property of bone marrow mesenchymal stem cells in nanofibrous vascular grafts, Proc. Natl. Acad. Sci. U. S. A., 2007, 104, 11915-11920.

36 J. Stitzel, L. Liu, S. J. Lee, M. Komura, J. Berry, S. Soker, et al., Controlled fabrication of a biological vascular substitute, Biomaterials, 2006, 27, 1088-1094.

37 X. Qiu, B. Li-Ping Lee, X. Ning, N. Murthy, N. Dong and S. Li, End-point immobilization of heparin on plasma-treated surface of electrospun polycarbonate-urethane vascular graft, Acta Biomater., 2017, 51, 138-147.

38 W. Cha and M. E. Meyerhoff, Catalytic generation of nitric oxide from S-nitrosothiols using immobilized organoselenium species, Biomaterials, 2007, 28, 19-27.

39 T. Koch, E. Suenson, U. Henriksen and O. Buchardt, The oxidative cleavability of protein cross-linking reagents containing organoselenium bridges, Bioconjugate Chem., 1990, 1, 296-304.

40 L. Heikal, G. P. Martin and L. A. Dailey, Characterisation of the decomposition behaviour of S-nitrosoglutathione and a new class of analogues: S-nitrosophytochelatins, Nitric Oxide, 2009, 20, 157-165.

41 P. N. Coneski, P. A. Fulmer, S. L. Giles and J. H. Wynne, Lyotropic self-assembly in electrospun biocidal polyurethane nanofibers regulates antimicrobial efficacy, Polymer, 2014, 55, 495-504.

42 L. M. He, S. Tang, M. P. Prabhakaran, S. Liao, L. L. Tian, Y. M. Zhang, et al., Surface Modification of PLLA Nanoscaffolds with Laminin Multilayer by LbL Assembly for
Enhancing Neurite Outgrowth, Macromol. Biosci., 2013, 13, 1601-1609.

43 W. Yuan, L. Huang, Q. Zhou and G. Shi, Ultrasensitive and selective nitrogen dioxide sensor based on self-assembled graphene/polymer composite nanofibers, ACS Appl. Mater. Interfaces, 2014, 6, 17003-17008.

44 J. M. Deitzel, J. Kleinmeyer, D. Harris and N. C. B. Tan, The effect of processing variables on the morphology of electrospun nanofibers and textiles, Polymer, 2001, 42, 261-272.

45 P. B. van Wachem, T. Beugeling, J. Feijen, A. Bantjes, J. P. Detmers and W. G. van Aken, Interaction of cultured human endothelial cells with polymeric surfaces of different wettabilities, Biomaterials, 1985, 6, 403-408.

46 P. B. van Wachem, A. H. Hogt, T. Beugeling, J. Feijen, A. Bantjes, J. P. Detmers, et al., Adhesion of cultured human endothelial cells onto methacrylate polymers with varying surface wettability and charge, Biomaterials, 1987, 8, 323-328.

47 M. Q. Wang, Y. Wang, Y. J. Chen and H. C. Gu, Improving endothelialization on $316 \mathrm{~L}$ stainless steel through wettability controllable coating by sol-gel technology, Appl. Surf. Sci., 2013, 268, 73-78.

$48 \mathrm{Y}$. Arima and H. Iwata, Effect of wettability and surface functional groups on protein adsorption and cell adhesion using well-defined mixed self-assembled monolayers, Biomaterials, 2007, 28, 3074-3082.

49 S. A. Sell, M. J. McClure, C. P. Barnes, D. C. Knapp, B. H. Walpoth, D. G. Simpson, et al., Electrospun polydioxanone-elastin blends: potential for bioresorbable vascular grafts, Biomed. Mater., 2006, 1, 72-80.

50 R. Sasisekharan and G. Venkataraman, Heparin and heparan sulfate: biosynthesis, structure and function, Curr. Opin. Chem. Biol., 2000, 4, 626-631.

51 J. X. Wu, H. W. Zhu, X. Chen, J. L. Wei, X. F. Zhang and M. Y. Xu, Inducible nitric oxide synthase inhibition reverses pulmonary arterial dysfunction in lung transplantation, Inflammation Res., 2014, 63, 609-618.

52 S. L. Chen, J. A. Jones, Y. G. Xu, H. Y. Low, J. M. Anderson and K. W. Leong, Characterization of topographical effects on macrophage behavior in a foreign body response model, Biomaterials, 2010, 31, 3479-3491.

53 E. Saino, M. L. Focarete, C. Gualandi, E. Emanuele, A. I. Cornaglia, M. Imbriani, et al., Effect of Electrospun Fiber Diameter and Alignment on Macrophage Activation and Secretion of Proinflammatory Cytokines and Chemokines, Biomacromolecules, 2011, 12, 1900-1911.

54 W. G. Brodbeck, M. S. Shive, E. Colton, Y. Nakayama, T. Matsuda and J. M. Anderson, Influence of biomaterial surface chemistry on the apoptosis of adherent cells, $J$. Biomed. Mater. Res., 2001, 55, 661-668.

55 Q. Zhao, N. Topham, J. M. Anderson, A. Hiltner, G. Lodoen and C. R. Payet, Foreign-body giant cells and polyurethane biostability: in vivo correlation of cell adhesion and surface cracking, J. Biomed. Mater. Res., 1991, 25, 177-183.

56 A. A. Khorana, A. Sahni, O. D. Altland and C. W. Francis, Heparin inhibition of endothelial cell proliferation and 
organization is dependent on molecular weight, Arterioscler., Thromb., Vasc. Biol., 2003, 23, 2110-2115.

57 C. B. Harrison, G. R. Drummond, C. G. Sobey and S. Selemidis, Evidence that nitric oxide inhibits vascular inflammation and superoxide production via a p47phoxdependent mechanism in mice, Clin. Exp. Pharmacol. Physiol., 2010, 37, 429-434.

58 C. Napoli, F. de Nigris, S. Williams-Ignarro, O. Pignalosa, V. Sica and L. J. Ignarro, Nitric oxide and atherosclerosis: an update, Nitric Oxide, 2006, 15, 265-279.

59 J. J. Xue, M. He, H. Liu, Y. Z. Niu, A. Crawford, P. D. Coates, et al., Drug loaded homogeneous electrospun PCL/gelatin hybrid nanofiber structures for anti-infective tissue regeneration membranes, Biomaterials, 2014, 35, 9395-9405.
60 A. Weigert and B. Brune, Nitric oxide, apoptosis and macrophage polarization during tumor progression, Nitric Oxide, 2008, 19, 95-102.

61 W. J. Lee, S. Tateya, A. M. Cheng, N. Rizzo-DeLeon, N. F. Wang, P. Handa, et al., M2 Macrophage Polarization Mediates Anti-inflammatory Effects of Endothelial Nitric Oxide Signaling, Diabetes, 2015, 64, 2836-2846.

62 D. M. Mosser and J. P. Edwards, Exploring the full spectrum of macrophage activation, Nat. Rev. Immunol., 2008, 8, 958969.

63 F. O. Martinez, A. Sica, A. Mantovani and M. Locati, Macrophage activation and polarization, Front. Biosci., Landmark Ed., 2008, 13, 453-461. 